\title{
BIOLOGIA FLORAL DE Virola surinamensis (ROL.) WARB. (MYRISTICACEAE)1
}

\author{
Mario Augusto Gonçalves Jardim² e Cléo Gomes da Mota ${ }^{3}$
}

\begin{abstract}
RESUMO - Neste artigo são apresentadas informações sobre a biologia floral de Virola surinamensis (Rol.) Warb. (Myristicaceae), espécie florestal dióica de relevante importância econômica na região amazônica. O estudo foi realizado em uma área de várzea próximo à bacia do igarapé Murutucum, lado direito do rio Guamá, localizada no Campus da Faculdade de Ciências Agrárias do Pará, na cidade de Belém, Estado do Pará, no período de janeiro a dezembro de 2001. Avaliou-se a biologia floral desde o aparecimento dos botões florais até a senescência das flores estaminadas, bem como a formação de frutos nas flores pistiladas. Testes bioquímicos foram aplicados para verificação de odor, pigmentos, osmóforos e receptividade do estigma. A observação no comportamento dos visitantes florais foi realizada durante o período diurno, registrando-se os horários de visitas, tempo de permanência na flor e frequiência; alguns indivíduos foram coletados com rede entomológica e identificados no Departamento de Zoologia do Museu Paraense Emílio Goeldi. A antese ocorreu entre 6 e $16 \mathrm{~h}$ nas flores estaminadas e entre 8 e $16 \mathrm{~h}$ nas flores pistiladas; a presença de odor foi constatada apenas nas flores estaminadas, enquanto os pigmentos e osmóforos foram encontrados em ambas as flores; o estigma mostrou-se receptivo no período entre 12 e $14 \mathrm{~h}$. Os insetos da ordem diptera foram os visitantes mais freqüentes nas flores estaminadas e pistiladas e as espécies Copestylum sp. e Erystalys sp., as responsáveis pela polinização.
\end{abstract}

Palavras-chave: Ecologia da polinização, testes bioquímicos e visitantes florais.

\section{Virola surinamensis (ROL.) WARB. (MYRISTICACEAE)FLORAL BIOLOGY}

\begin{abstract}
Information was obtained on the floral biology of Virola surinamensis (Rol.) Warb. (Myristicaceae), a dioecious arboreal species of great importance for the Amazon region economy. The study was carried out in the floodplain area near the Murucutu stream, on the right side of the Guama River, at the Universidade Federal Rural da Amazônia-UFRA, Belém-Pará, from January to December 2001. Floral biology was assessed from floral bud phase to senescence stage in male flowers and fruit formation in female flowers. Biochemical tests were carried out to verify odor, pigment, osmophore pigments and stigma receptivity. Floral visitors were observed during the diurnal period and visiting time, flower permanence time and frequency were recorded. Some individuals were collected with entomological net and identified by the Zoology Department of the Emílio Goeldi Paranaense Museum. Anthesis occurred between 06:00 a.m. and 4:00 p.m. in the staminate flowers, and between 8:00 a.m. and 4:00 p.m. for the pistilate flowers. Odor was reported only in the staminate flowers and osmophore pigments in both flowers types. The stigma showed to be receptive between 12:00 and 2:00 p.m. Diptera order insects were the most frequent visitors on the staminate and pistilate flowers. The Copestylum sp. and Erystalys sp. were responsible for pollination.
\end{abstract}

Keywords: Pollination ecology, biochemistry tests and floral visitors.

\footnotetext{
${ }^{1}$ Recebido em 20.12.2006 e aceito para publicação em 02.07.2007.

${ }^{2}$ Museu Paraense Emílio Goeldi- Coordenação de Botãnica. Pesquisador MCT/Bolsista produtividade em Pesquisa - CNPq. . E-mail:<jardim@museu-goeldi.br>.

${ }^{3}$ Universidade Federal Rural da Amazônia - Mestre em Agronomia. E-mail: <motacleo@ hotmail.com.br>.
} 


\section{INTRODUÇÃO}

A biologia floral é importante para o entendimento da interação planta-animal e, principalmente, para o sistema de cruzamento das plantas. As interações e os sistemas de cruzamento são considerados indicadores biológicos por contribuírem nos planos de conservação e manejo e no entendimento do fluxo gênico das populações (JARDIM, 1991). Os estudos sobre biologia floral visam ao melhoramento, conservação genética e produção de sementes e devem ser baseados no conhecimento do modo de reprodução da espécie (GUSSON et al., 2006). Ao mesmo tempo auxiliam o entendimento do fluxo gênico e o sistema de reprodução (BAWA, 1974, 1980). Autores como Bawa (1974), Opler e Bawa (1978), Bawa e Beach (1981), Bullock e Bawa (1981), Bullock (1982), Armstrong e Drummond (1986), Jardim (1991), Pinã-Rodrigues et al. (1993), Pombal e Morellatto (1995), Jardim e Macambira (1997), Venturieri e Silva (1997), Maués et al. (1999) e Venturieri et al. (1999) têm estudado a ecologia de polinização em espécies florestais hermafroditas e monóicas de regiões tropicais, enquanto Bawa (1980) e Bullock (1982) relataram estudos sobre a polinização de plantas dióicas. Segundo esses dois últimos autores, as plantas dióicas estabelecem sincronismos eficazes no florescimento das plantas masculinas e femininas e na oferta de recursos florais para auxiliar a sua reprodução, por intermédio dos polinizadores, portanto, eles autores consideram o dioicismo um aspecto extremamente evoluído das espécies.

Virola surinamensis (Rol.) Warb. é uma espécie dióica encontrada em extensas populações nos ecossistemas de várzea e igapó na Amazônia, principalmente na região do estuário. Apresenta valor comercial madeireiro para produção de compensados, laminados, e fabricação de cabos de vassoura. No ano de 1994, ocupou o segundo lugar em exportação no Estado do Pará (ANDERSON et al., 1994). Considerando o valor comercial e de preservação da espécie é que este trabalho teve como objetivo obter informações sobre a biologia floral da espécie, visando contribuir com indicadores de conservação e manejo, a partir do conhecimento da estrutura e funcionalidade das flores e os visitantes florais associados à polinização.

\section{MATERIAL E MÉTODOS}

O estudo foi desenvolvido em uma área de várzea, próxima à bacia do igarapé Murutucum, lado direito do rio Guamá, localizada no Campus da Faculdade de Ciências Agrárias do Pará, com coordenadas geográficas de $01^{\circ} 27^{\prime} 25^{\prime \prime}$ e $01^{\circ} 27^{\prime} 39$ " latitude S e $48^{\circ} 26^{\prime} 36^{\prime}$ " e $48^{\circ} 26^{\prime} 48^{\prime \prime}$ de longitude WGr, sendo a maior altitude da área de $41 \mathrm{~m}$. A classificação climática de acordo com Köppen é do tipo Afi. A pluviosidade média anual é de $2.900 \mathrm{~mm}$ e temperatura média anual de $25,9^{\circ} \mathrm{C}$, variando entre 21 e $31^{\circ} \mathrm{C}$. A umidade relativa está em torno de $84 \%$, e ocorrem 2.219 h de insolação anual. Os meses de maior pluviosidade se estendem de janeiro a março. O ambiente é composto por vegetação secundária bastante alterada por intervenções antrópicas e pela pecuária.

As observações da biologia floral foram efetuadas com o auxílio de duas torres metálicas de $25 \mathrm{~m}$ de altura alocadas próximas a quatro árvores masculinas e quatro femininas, em fase de floração. O período de observação da floração nos indivíduos masculinos foi de janeiro a junho de 2001, compreendendo desde a formação do botão floral até a abscisão total das flores. Nas plantas femininas, esse período foi de julho a dezembro de 2001 e compreendeu desde a formação do botão floral até a fase inicial da formação dos frutos. Foram registrados os horários de antese, mudanças florais (cor, odor, presença de pólen e néctar) e abscisão da flor nos seguintes horários: $10 \mathrm{~h}, 12 \mathrm{~h}, 14 \mathrm{~h}, 16 \mathrm{~h} \mathrm{e}$ $18 \mathrm{~h}$ durante 10 dias consecutivos.

Os testes bioquímicos foram aplicados para verificação de odores, pigmentos, osmóforos e receptividade do estigma, segundo métodos usados por Dafni (1992), Jardim (1991) e Venturieri e Silva (1997). A classificação de odores foi feita colocando-se 10 flores de cada sexo em recipiente de vidro hermeticamente fechado em diferentes horários: $8 ; 12 ; 14 ; 16 ; 18$ e 22 h e avaliando-se conforme a seguinte classificação: $0=$ sem odor, $1=$ odor percebido somente com insistência, $2=$ odor moderado percebido com dificuldade e $3=$ odor intenso percebido prontamente (VENTURIERI e SILVA, 1997). Para a detecção dos pigmentos foi utilizado um béquer com $1 \mathrm{~mL}$ de hidróxido de amônia 24\%. As flores foram colocadas em um pequeno crivo acoplado ao béquer e coberto com papel laminado por $10 \mathrm{~min}$, com finalidade de expor as partes florais aos gases. As alterações ocorrentes foram anotadas conforme Venturieri e Silva (1997). Para avaliar a presença de osmóforos, foi utilizada a metodologia de Vogel (1963), com a imersão das flores em solução de vermelho neutro (1:1000) em diferentes intervalos de tempo (10', 30', $1 \mathrm{~h} \mathrm{e} 2 \mathrm{~h}$ ). 
Posteriormente, as flores foram lavadas com água para retirar o excedente, e as regiões coradas foram anotadas. Para a receptividade do estigma foram observadas 20 flores em diferentes horários após a antese (CRESTANA, 1993). A reação foi quantificada pelo número de bolhas de ar formadas em 1 min.

As observações sobre o comportamento dos visitantes florais foram realizadas de janeiro a dezembro de 2001, durante o período diurno, classificando-os em ocasional, pilhador, predador e provável polinizador. Registraram-se os horários de visitas, tempo de permanência na flor e freqüência (número de indivíduos/ flor). Alguns indivíduos foram capturados com rede entomológica, acondicionados em álcool $70 \% \mathrm{e}$ identificados na Coordenação de Zoologia do Museu Paraense Emílio Goeldi.

\section{RESULTADOS E DISCUSSÃO}

\subsection{Resultados}

Nas flores estaminadas, a antese ocorreu nas primeiras horas do dia $(6 \mathrm{~h})$ até o final da tarde, e o maior pico de abertura ocorreu entre 12 e $16 \mathrm{~h}$. No momento da antese, os grãos de pólen já se encontravam expostos nas aberturas longitudinais das anteras. Seis horas após a antese, as flores passaram a apresentar coloração marrom-escuro, e após 24 h observou-se o início da senescência floral. As bordas das pétalas adquiriram cor amarelo-escura, e isso ocorreu concomitantemente com o início da abscisão. As flores estaminadas foram consideradas efêmeras, pois permanecem pouco mais de $24 \mathrm{~h}$ inseridas na inflorescência.

Nas flores pistiladas, a antese ocorreu durante o dia, desde as $8 \mathrm{~h}$ até o final da tarde. Entretanto, o maior número de flores em antese aconteceu entre 9 e $16 \mathrm{~h}$. No período das 12 às $16 \mathrm{~h}$, constatou-se maior número de flores abertas. Apresentaram odor suave e adocicado, estigma amarelo e receptivo com lóbulos estigmáticos fechados, mas com a presença de exsudato. Os lóbulos estigmáticos abriram-se no decorrer do dia, permanecendo cerca de $48 \mathrm{~h}$ abertos. A posição dos lóbulos estigmáticos (aberto ou fechado) demonstrou maior porcentual de flores com estigma aberto no período da tarde (Figura 1), enquanto a produção de exsudato foi maior pela manhã (Figura 2).

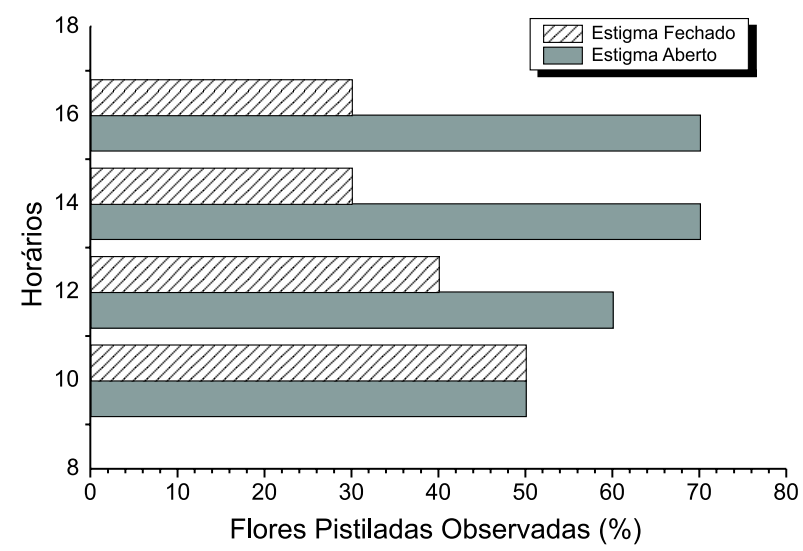

Figura 1 - Posição do estigma (aberto e fechado) de Virola surinamensis (Rol.) Warb. expresso em porcentagem por horário de observação.

Figure 1 -Position of stigma (open and closed) of Virola surinamensis (Rol.) Warb., expressed in percentage per hours of observation.

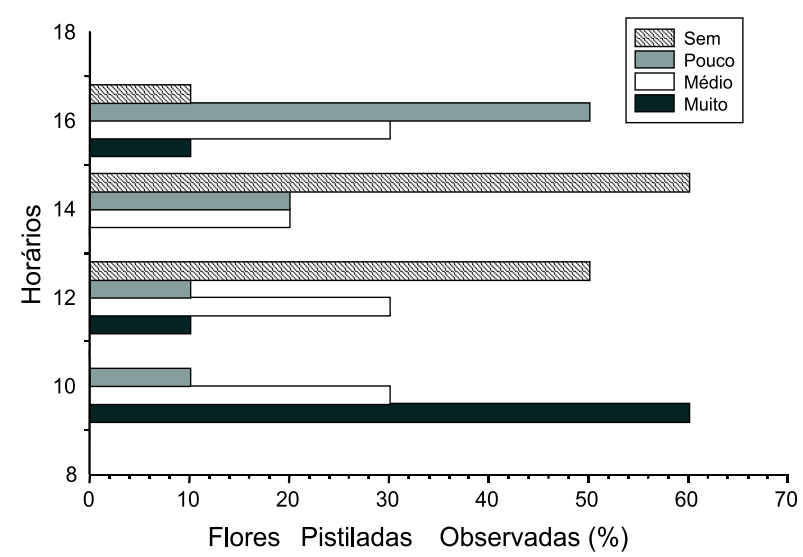

Figura 2 - Porcentual de flores pistiladas de Virola surinamensis (Rol.) Warb. com exsudato expresso em percentagem por horário de observação.

Figure 2 - Percentage of pistilated flowers of Virola surinamensis (Rol.) Warb with exudate expressed in percentage per hours of observation.

As mudanças florais iniciaram-se $48 \mathrm{~h}$ após a antese, quando o estigma foi adquirindo coloração marrom-escura das bordas para o centro. Após $72 \mathrm{~h}$ as flores foram adquirindo tonalidade amarelo-escura, e até o $7^{\circ}$ dia de abertura as bordas das pétalas começaram a escurecer, iniciando o processo de abscisão das flores que não foram polinizadas. As flores fecundadas permaneceram com dilatação do ovário. Após o $15^{\circ}$ dia continuaram inseridas nas inflorescências apenas as flores que se encontravam em processo de formação do fruto.

R. Árvore, Viçosa-MG, v.31, n.6, p.1155-1162, 2007 
Quanto aos testes bioquímicos, as flores masculinas e femininas apresentaram odor perceptível suave e adocicado; mudança de pigmentação de amarelo claro para amarelo escuro, indicando a presença de flavonóides; ocorrência de osmóforos nas pétalas e o estigma receptivo principalmente no período das 12 às $14 \mathrm{~h}$.

Estão listados na Tabela 1 os espécimes das ordens Diptera, Hymenoptera, Coleoptera e Homóptera, que visitaram as flores masculinas e femininas e os respectivos comportamentos. Os insetos da Ordem Diptera, família Syrphidae, visitaram flores estaminadas e pistiladas durante todo o dia, principalmente nos horários entre 9 e 17 h. Nas flores estaminadas, coletouse pólen e nas pistiladas, o néctar. Pelo comportamento de forrageamento, foram classificados como polinizadores ocasionais.

Insetos da Ordem Hymenoptera foram freqüentes nas flores pistiladas no período das $8 \mathrm{~h}$ às $17 \mathrm{~h}$, cujo comportamento não demonstrou nenhuma relação com o transporte de pólen, pois só visitaram flores pistiladas como pilhadores e visitantes ocasionais. Os visitantes das Ordens Coleoptera e Homoptera tiveram baixa freqüência tanto nas flores estaminadas quanto nas pistiladas. Consideraram-se, mediante a baixa freqüência dos indivíduos e a periodicidade nas flores classificados como pilhadores de pólen e néctar, predadores de partes florais ou visitantes ocasionais.

\subsection{Discussão}

De acordo com Endress (1994), os movimentos dos lóbulos estigmáticos têm função ecológica e afetam a hercogamia agindo como um sinal visual para os polinizadores. Em plantas dióicas, o florescimento apresenta maior tempo de duração nos indivíduos masculinos e femininos (BAWA, 1983; GORI, 1983; NEWSTRON et al., 1994). Segundo Melampy e Howe (1977) e Bullock e Bawa (1981), as árvores dióicas masculinas iniciam a floração mais cedo e permanecem mais tempo em relação às femininas. Para Willson (1979), Bawa (1980), Stephenson e Bertin (1983) e Bawa (1983), nas espécies dióicas tropicais as plantas masculinas investem mais na produção de flores do que as femininas.

O florescimento masculino prolongado e antecipado também pode ser importante na atração e manutenção do número de polinizadores necessários para o aumento do sucesso reprodutivo. Porém, essas diferenças entre as fases florais têm sido atribuídas ao alto custo fisiológico da produção de frutos, resultando em aumento proporcional na alocação de recursos para a reprodução feminina (LLOYD e WEBB, 1977; DELPHe MEAGHER, 1995). Para Newstron et al. (1994), nas espécies dióicas existem diferenças no modelo de floração entre plantas masculinas e femininas. Os resultados encontrados em V.surinamensis a respeito do maior tempo de maturação das flores masculinas em relação às flores femininas estão de acordo com aqueles encontrados nas espécies do gênero Virola por Mantovani et al. (2003), Willson (1979), Bawa (1980), Stephenson e Bertin (1983), Bawa (1983) e Newstron et al. (1994). Possivelmente, as flores masculinas teriam a função de atrair maior número de visitantes florais com maior oferta de alimento em maior espaço de tempo.

Tabela 1 - Visitantes florais de Virola surinamensis (Rol.) Warb. por Ordem, Família, Espécie e comportamento, no período de janeiro a dezembro de 2001

Table 1 - Floral visitors of Virola surinamensis (Rol.) Warb. by Order, Family, Species and behavior, from January to December 2001

\begin{tabular}{clll}
\hline Ordem & Família & Espécie & Comportamento \\
\hline Diptera & Syrphidae & Copestylum sp. & Polinizador \\
& Syrphidae & Erystalys sp. & Polinizador \\
Hymenoptera & Apidae & Appis mellifera & Pilhador \\
& Apidae & Trigona recursa & Pilhador \\
& Apidae & Trigona spinipes & Pilhador \\
& Halictidae & Augochloropsis ilustris & Ocasional \\
& Halictidae & Halictus nigromarginatus & Pilhador \\
& Formicidae & Camponotus femoratus & Ocasional \\
Meliponidae & Melipona ponclicoliis & Ocasional \\
Coleoptera & Xylocopidae & Xylocopa neoxylocopa & Pilhador \\
& Tenebrionidae & Epitragini sp. & Pilhador \\
Carabidae & Lebia sp. & Ocasional \\
\hline
\end{tabular}

R. Árvore, Viçosa-MG, v.31, n.6, p.1155-1162, 2007 
Resultados semelhantes foram encontrados por Bullock (1982) na Costa Rica, em seus estudos a respeito da estrutura populacional e do padrão de distribuição espacial da espécie dióica Campsoneura sprucei (A.DC.) Warb., que constatou comportamento diferenciado entre árvores masculinas e femininas em relação ao grau de florescimento. Piña-Rodrigues et al. (1993) citaram que em $V$.surinamensis o padrão de florescimento é mais intenso durante o ano. Na espécie dióica Genipa americana L., a época de florescimento, o horário de antese e tempo de duração das flores pistiladas e estaminadas são diferenciados, e o assincronismo beneficia o sistema de cruzamento (CRESTANA, 1993). Frankie et al. (1983), estudando espécies arbóreas da Costa Rica, observaram que todas as espécies dióicas apresentaram flores estaminadas e pistiladas com diferenças temporais na produção de recursos florais. Segundo esses autores, a ausência de sincronismo no fluxo de néctar entre flores de sexos diferentes facilita o movimento dos polinizadores. Bawa e Beach (1981), correlacionando modos de polinização, sistemas reprodutivos e fenologia de espécies arbóreas tropicais dióicas, e constataram que as árvores masculinas apresentam antecipação de horário de antese com maior duração no período de floração e, em alguns casos, com maior freqüência de florescimento em comparação com as plantas femininas.

Para Bawa (1983), nas plantas dióicas é comum encontrar diferenças na duração da floração, pois, se de fato o grau de fecundação floral é determinado principalmente pela floração masculina, seria necessário o prolongamento da floração feminina para a produção de frutos. No entanto, extenso período de floração feminina pode ser encurtado se ocorrer limitação nos recursos florais para polinizadores. Os resultados obtidos em V.surinamensis concordam com essa afirmativa, pois as flores masculinas funcionam somente como doadoras de pólen e investem, principalmente, na quantidade com flores pequenas de curta longevidade. Entretanto, as flores femininas investem mais na qualidade da fertilização e mais tempo na inflorescência. Para Ashman e Schoen (1996), a longevidade floral nas angiospermas é um caráter que se reflete na adaptação da planta a diversas condições bióticas e abióticas, fazendo que ocorram períodos longos e curtos no florescimento e, ao mesmo tempo, sincronizando a produção de recursos florais para agentes polinizadores.
A principal recompensa de $V$. surinamensis ofertada aos visitantes nas flores estaminadas foi o pólen. Entretanto, observaram-se insetos em botões florais e pétalas em ambos os sexos, em que os testes bioquímicos revelaram a presença de osmóforos. Nas flores pistiladas, o néctar foi a principal recompensa. Não se observou produção de néctar nas flores estaminadas. PiñaRodrigues et al. (1993) afirmaram que as flores pistiladas de V. surinamensis geralmente apresentam baixa quantidade de néctar, geralmente nas primeiras horas do dia. O maior número de agentes visitantes foi observado nas flores masculinas, dentre esses os dípteros das famílias Drosophilidae, Syrphidae e Muscidae, o que permitiu considerar a importância dos insetos dessas famílias como prováveis polinizadores.

De acordo com Faegri e van der Pijl (1979), a eficiência na polinização realizada por pequenos dípteros é limitada pelo seu tamanho, pois carregam poucos grãos de pólen, mas a capacidade de transporte pode ser compensada pela frequiência de visitas. De acordo com Kevan (1983), os Syrphideos e outros dípteros com probóscide longa são hábeis para explorar flores tubulares. Segundo Proctor et al. (1996), os dípteros são atraídos por flores de cores amarela, branca, parda e púrpura semelhantes à cor de excremento ou material em decomposição. Entretanto, o odor adocicado pode guiar os insetos na busca desse recurso.

É interessante ressaltar a estratégia de atração utilizada por Virola surinamensis. As plantas masculinas apresentam grande investimento na produção de flores com maior visibilidade, indicando que essas árvores podem atrair insetos a grandes distâncias. Enquanto as plantas femininas menos vistosas (menor número de flores) também podem atrair insetos, principalmente pela similaridade às flores e inflorescências masculinas e pelo seu odor característico, comum aos dois sexos. Dessa forma, supõe-se que as flores femininas são polinizadas por engano. Nesse contexto, o odor deve possuir primordial atuação na atração dos polinizadores. Segundo Endress (1994), existe grande número de espécies de Magnoliidae que são polinizadas por dípteros. Assim, podem-se sugerir estudos futuros que testem, de maneira apropriada, a hipótese de que a Virola surinamensis é efetivamente polinizada por dípteros e, ou, existe co-evolução entre dípteros e Myristicaceae.

Pombal e Morellatto (1995), estudando as características florais e a polinização de Dendropanax

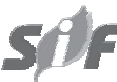

R. Árvore, Viçosa-MG, v.31, n.6, p.1155-1162, 2007 
cuneatus (DC.) Decne. \& Planch. (Araliaceae) em floresta semidecídua no sudoeste do Brasil, observaram que as flores foram visitadas, sobretudo, por dípteros. Porém, os muscídeos foram os mais importantes, sendo Morellia humeralis e $M$. dendropanacis considerados os principais polinizadores. Venturieri et al. (1999), estudando a ecologia reprodutiva do taxi-branco (Sclerolobium paniculatum Vogel), verificaram que os dípteros das famílias Syrphidae e Stratiomiidae foram os polinizadores ocasionais. Segundo Percy e Cronk (1997), na espécie dióica Nesohedyotis arborea (Hook.f.) Bremek. a polinização foi realizada por pequenos Syrfídeos. Para Howe (1990), V. surinamensis apresenta padrão de distribuição agregada e síndrome de polinização entomófila, com maior freqüência de Syrfídeos, Drosofilideos e Muscideos, considerados polinizadores de curta distância de vôo.

Os dados obtidos de $V$. surinamensis em relação à morfologia e coloração das flores são característicos de plantas dióicas. Esses dados coincidem com os resultados de Opler e Bawa (1978), Bawa (1980), Bawa e Beach (1981) e Bawa (1983), quando afirmaram que o dioicismo está relacionado às plantas com flores pequenas e de coloração branca, amarela ou verde, cuja morfologia não especializada atrai variedades de insetos pequenos e generalistas. Para Kevan (1983) e Endress (1994), grande variedade de insetos não especializados preferem flores amarelas, enquanto para Proctor et al (1996) a cor da flor pode estar associada à localização ou exposição do recurso floral. Dessa forma, as flores de cor amarela poderiam atrair insetos generalistas que visitam flores abertas. Comparando os resultados com o descrito por Desai et al. (1994), observou-se que $V$. surinamensis tem características similares às de Mangifera indica L. (Anacardiaceae), principalmente quanto ao tipo de inflorescência e insetos visitantes, além de possuir inflorescências do tipo panículas cônicas e antese diurna entre 7 e $11 \mathrm{~h}$.

\section{CONCLUSÕES}

As características florais de Virola surinamensis quanto ao tamanho e cor das flores associadas ao assincronismo das flores estaminadas e pistiladas favorecem o dioicismo. Tais características estão diretamente relacionadas às ofertas de recursos florais para diversos agentes visitantes. Todavia, estabelecem um grau de especificidade com as espécies Copestylum sp e Erystalys sp. como principais polinizadores. Em relação a outros estudos com plantas dióicas, os resultados apontaram que existe certo grau de especificidade entre planta $\mathrm{x}$ inseto quanto ao tamanho das flores e à produção de néctar.

\section{REFERÊNCIAS}

ANDERSON, A. B.; MACEDO, D. S.; MOUSASTICHOSHVILY. I. C. Impactos ecológicos e sócio-econômicos da exploração seletiva de Virola no estuário amazônico: implicações para politícas florestais brasileiras. Relatório final, WWF. Rio de Janeiro: 1994. 76p.

ARMSTRONG, J. E.; IRVINE, A. K. Flowering, sex ratio, pollen-ovulo, fruit set and reproductive effort of a dioecius tree, two different rain forest community. American Journal of Botany, v.76, n.1, p.74-85, 1989.

ASHMAN, Tia-Lynn; SCHOEN, D. J. Floral longevity: Fitness consequences and resources cost. In: LLOYD, D.G.; BARRETT, S.C.H. (Ed.). Floral biology: Studies on floral evolution in animal-pollinated plants. New York: Chapman \& Hall, 1996. p.410.

BAWA, K. S. Breeding systems of tree species of a lowland tropical community. Evolution, v.28, n.1, p.15-39, 1974.

BAWA, K. S. Evolution of dioecy in flowering plants. Annual Review of Ecology and Systematics, v.11,.p.15-39, 1980.

BAWA, K. S.; BEACH, J. H. Evolution of sexual systems in flowering plants. Annals of the Missouri Botanical Garden., v.68, p.254-274, 1981 .

BAWA, K. S. Patterns of flowering in tropical plants. In: JONES, C. E.; LITTLE, R. J. (Ed.). Handbook of experimental pollination biology. New York: Scientific and Academic, 1983. p.394-410.

BULLOCK, S. H. Population structure and reproduction in the neotropical dioecious Campsoneura sprucei (A. DC.). Oecologia, v.55, p.494-1504, 1982. 
BULLOCK, S. H.; BAWA, K. S. Sexual dimorphism and the annual flowering pattern in Jacaratia dolichaula (D. Smith) Woodson (Caricaceae) in a Costa Rican Forest. Ecology, v.62, p.1494-1505, 1981.

CRESTANA C. S. M. Biologia da reprodução de Genipa americana $L$. (Rubiaceae) na Estação Ecológica de Moji-Guaçu Estado de São Paulo. 1993. p.103 Tese (Doutorado em Botânica) Universidade Estadual Paulista, Rio Claro, 1993.

DAFNI, A. Pollination ecology. Oxford: Oxford University Press, 1992. 250p.

DELPH, L. F; MEAGHER, T. R. Sexual dimorphism masks life history trade-offs in the dioecious plant Silene Latifolia. Ecology, v.76, n.3, p.775-785, 1995.

DESAI, U. T. et al. Floral biology of mango hybrid Sai-Sugandh. Recent Horticulture, v.1, n.1, p.11-13, 1994.

ENDRESS, P. K. Diversity and evolutionary biology of tropical flowers. Cambridge: Cambridge University Press, 1994. 511p.

FAEGRI, K.; van der PILJ, L. The principles of pollination ecology. 3.ed. Oxford: Pergamon Press, 1979. 244p.

FRANKIE, G. W. et al. Caracteristics and organization of the large bee pollination system in the Costa Rican dry Forest. In: JONES, C. E.; LITTLE, R. J. (Ed.). Handbook of experimental pollination biology. New York: Scientific and Academic, 1983. p.411-448.

GORI, D. F. Post-pollination phenomena and adaptive floral changes. In: JONES, C. E.; LITTLE, R. J. (Ed.). Handbook of experimental pollination biology. New York: Scientific and Academic Editions, 1983. p.31-49.

GUSSON, E.; SEBBENN,A. M.; KAGEYAMA, P. Y. Sistema de reprodução em populações de Eschweilera ovata (Cambess.) Miers. Revista Árvore, v.30, n.4, p.491-502, 2006.

HOWE, H. F. Survival and growth of juvenile Virola surinamensis in Panama: Effects of herbivory and canopy closure. Jornal of Tropical Ecology, v.6, n.1, p.259-280, 1990.
JARDIM, M. A. G. Aspectos da biologia reprodutiva de uma população natural de açaizeiro (Euterpe oleracea Mart.) no Estuário Amazônico. 1991. 91p. Dissertação (Mestrado em Ciências Florestais) - Escola Superior de Agricultura Luiz de Queiroz, Piracicaba, 1991.

JARDIM, M. A. G.; MACAMBIRA, M. L. J. Identificação dos insetos visitantes de inflorescências da palmeira inajá (Maximiliana maripa L.). Boletim do Museu Paranaense Emílio Goeldi, Série Zoologia, v.13, n.1, p.85-94, 1997.

KEVAN, P. G. Floral colors through the insect eyes: What they are and what they mean. In: JONES, C. E.; LITTLE, R. J. (Ed.). Handbook of experimental pollination biology. New York: Scientific and Academic, 1983. p.3-30.

LLOYD, D. G.; WEBB, C. J. Secondary sex characteres in plants. Botanical Review, v.43, p.177-216, 1977.

MANTOVANI, M. et al. Fenologia reprodutiva de espécies arbóreas em uma formação secundária da floresta Atlântica. Revista Árvore, v.27, n.4, p.451-458, 2003.

MAUÉS, M.M. et al. Biologia de polinização do acapú (Vouacapoua americana Aubl.), Leguminosae, uma essência florestal Amazônica. In: SIMPÓSIO DE SILVICULTURA NA AMAZÔNIA ORIENTAL: CONTRIBUIÇÕES DO PROJETO EMBRAPA/DFID. Belém, Embrapa/CAPTU/DFID, 1999.309p.

MELAMPY, M. N.; HOWE, H. F. Sex ratio in the tropical tree Triplaris americana (Polygonaceae). Evolution, v.31, p.867-872, 1977.

NEWSTRON, L. E. et al. Diversity of long-term flowering paterns. In: MCDADE, L. A. et al. (Ed.). La selva: ecology and natural history of a neotropical rain forest. Chicago: Chicago University Press, 1994. p.35-46.

OPLER, P. A.; BAWA, K. S. Sex ratio in tropical forest trees. Evolution, v.32, n.4, p.812-821, 1978.

PERCY, D. M.; CRONK, Q. C. B. Conservation in relation to mating system in Nesohedyotis arborea (Rubiaceae), a rare endemic tree from St. Helena. Biological Conservation, v.80, n.2, p.135-145, 1997.

R. Árvore, Viçosa-MG, v.31, n.6, p.1155-1162, 2007 
PIÑA-RODRIGUES, F. C. M. et al. Ecologia da polinização de Virola surinamensis (Rol) Warb. (Myristicaceae) no estuário amazônico. In: CONGRESSO NACIONAL DE BOTÂNICA, 1993, São Luiz. Anais... São Luiz, Sociedade Botânica do Brasil, 1993. p.188

POMBAL, E. C. P.; MORELLATTO, P. C. Polinização por moscas em Dendropanax cuneatum Decne. \& Planch. (Araliaceae) em floresta semidecídua no sudeste do Brasil. Revista Brasileira de Botânica, v.18, n.2, p.157-162, 1995.

PROCTOR, M.; YEO, P.; LACK, A. The natural history of pollination. London: Harper Collins, 1996. 479p.

STEPHENSON, A. G.; BERTIN, R. I. Male competition, Female choice, and sexual selection in plant. In: REAL, L. (Ed.). Pollinization biology, Orlando: Academic Press, 1983.p.110-140.
VENTURIERI , G. A.; SILVA, M. B. Fenologia de floral do cacau-jacaré (Herrania mariae) Sterciliaceae. Boletim do Museu Paranaense Emílio Goeldi, Série Botânica, v.13, n.1, p.31-47, 1997.

VENTURIERI, G. C.; BRIENZA Jr., S.; NEVES, C. B. Ecologia reprodutiva do taxi-branco (Sclerolobium paniculatum var.paniculatum Voge1), Leguminosae. In: SIMPÓSIO DE SILVICULTURA NA AMAZÔNIA ORIENTAL: CONTRIBUIÇÕES DO PROJETO EMBRAPA/DFID. Belém: Embrapa/CAPTU/DFID, 1999. 309p.

VOGEL, S. Duftddrüsen in Dienste der Osmofhoren. Journal Botanik

Studieren, 10, p.600-763, 1963.

WILLSON, M. F. Sexual selection in plants. American Naturalist, v.113, p.777-790, 1979. 